\title{
The Role of Kelvin-Helmholtz Instability for Producing Loop-top Hard X-ray Sources in Solar Flares
}

\author{
Xia Fang, Ding Yuan, Chun Xia, Tom Van Doorsselaere, and Rony Keppens \\ Centre for mathematical Plasma Astrophysics, Department of Mathematics, KU Leuven, \\ Celestijnenlaan 200B, 3001 Leuven, Belgium
}

\begin{abstract}
We propose a model for the formation of loop-top hard X-ray (HXR) sources in solar flares through the Inverse Compton mechanism, scattering the surrounding soft X-ray (SXR) photons to higher energy HXR photons. We simulate the consequences of a flare-driven energy deposit in the upper chromosphere in the impulsive phase of single loop flares. The consequent chromosphere evaporation flows from both footpoints reach speeds up to hundreds of kilometers per second, and we demonstrate how this triggers Kelvin-Helmholtz instability (KHI) in the loop top, under asymmetric conditions. The KHI vortices further fragment the magnetic topology into multiple magnetic islands and current sheets, and the hot plasma within leads to a bright loop-top SXR source region. We argue that the magnetohydrodynamic turbulence that appears at the loop apex could be an efficient accelerator of non-thermal particles, which the island structures can trap at the loop-top. These accelerated non-thermal particles can upscatter the surrounding thermal SXR photons emitted by the extremely hot evaporated plasma to HXR photons.
\end{abstract}

Subject headings: magnetohydrodynamics(MHD) — Sun: corona — Sun: flares — Sun: $\mathrm{X}$-rays, gamma rays

\section{Introduction}

Solar flares, the most powerful events of solar activity, are thought to be the result of magnetic reconnection occuring in the corona and can release in excess of $10^{32}$ ergs of energy in a matter of minutes (Hudson 2011). A substantial fraction of the released energy is transported downwards along the magnetic loop by non-thermal particles (Brown 1971), or by thermal conduction (Fletcher et al. 2011) and/or Poynting flux (Fletcher \& Hudson 2008). The bulk of the non-thermal particle energy gets deposited in the chromosphere via Coulomb collisions (Brown 1971). This produces HXR emission via non-thermal bremsstrahlung primarily at the footpoints of the loops (Hoyng et al. 1981) and is referred to as the thick target model (Brown 1971). The overpressure resulting from the tremendous energy deposit drives an upward mass flow at a speed up to hundreds of kilometers per second, known as chromospheric evaporation. The affected coronal loops thereby get filled with 
hot plasma and these loops are then observed via thermal SXR emission (Veronig et al. 2005). Since the intensity of non-thermal bremsstrahlung depends on the ambient plasma density, HXR emission is usually too weak to occur in the corona, where the densities are generally much lower. Only in a few cases, coronal densities seem to be high enough such that HXR sources can be clearly observed in the corona (e.g. Veronig \& Brown 2004). Nevertheless, several examples clearly show HXR emission from coronal sources (Frost \& Dennis 1971; Hudson 1978; Masuda et al. 1994; Tomczak 2001; Krucker \& Lin 2008). Arguably the most famous example showed an impulsive non-thermal HXR source appearing above thermal SXR loops by Masuda et al. (1994), and this confirmed the overlying cusp geometry of the standard solar flare model (Shibata \& Magara 2011). However, although several somewhat similar events have been observed since (Tomczak 2001; Krucker \& Battaglia 2014), the Masuda event has not proven to be typical of solar flares in general. Most events with both SXR and HXR coronal sources show less separation $(\leq 3,500 \mathrm{~km})$ between the thermal SXR loops and the coronal HXR source, and many thermal events in a loop that fills by chromospheric evaporation even show co-spatial non-thermal emission (Tomczak 2001; Krucker \& Lin 2008).

Therefore, the emission mechanism of these rare loop-top HXR events might be different from the Masuda flare, which was interpreted as a partially thick target up in the corona, by injecting non-thermal particles into the cusp of a magnetic trap (Fletcher 1995). Korchak (1971) concluded that an Inverse Compton (IC) contribution might sometimes be significant, particularly in the low ambient density conditions relevant to these loop-top HXR events. With an ambient coronal number density of $10^{9} \mathrm{~cm}^{-3}$, Inverse Compton radiation eventually dominates over bremsstrahlung in the 10-100 keV photon energy range (Krucker et al. 2008). Furthermore, these bright, concentrated, loop-top coronal HXR sources show time variation in the order of tens of seconds to minutes and are most prominent during the rise of the thermal emission (Krucker et al. 2008). Therefore, the populations of loop-top non-thermal particles which possibly contribute to HXR emission by IC could be accelerated in turbulent plasma conditions, which also helps to trap particles (Turkmani et al. 2006; Krucker et al. 2008). In such cases, the accelerator is then believed to be co-spatial with the coronal HXR source.

In this paper, we propose a model which generates turbulence in the loop top by the KelvinHelmholtz instability (KHI). The KHI is triggered by the chromospheric evaporation flows from asymmetric flare energy deposition at both loop footpoints. KHI, produced when two fluid flows have a velocity shear across an interface or finite extent region (Chandrasekhar 1961), is able to form vortices which can trigger further fine-scale reconnection and turbulence in a magnetized plasma Keppens et al. (1999); Baty et al. (2003); Henri et al. (2013). KHI is well studied at fast-slow stream interfaces in a variety of solar structures (Heyvaerts \& Priest 1983; Andries \& Goossens 2001), and has even been observed directly in connection with a coronal mass ejection (Foullon et al. 2011) or in solar prominences (Berger et al. 2010). Here, we will demonstrate KHI in a single suddenly footpoint-heated loop.

The paper is then organized as follows: in $\S 2$ we describe the numerical setup; in $\S 3$ we show 
the results of the simulation and discuss the implications, and conclusions are drawn in $\S 4$.

\section{Computational Aspects}

\subsection{Initial Setup}

Our numerical setup, initial and boundary conditions follow the model in Fang et al. (2015), using a single-fluid magnetohydrodynamic (MHD) plasma description on a two-dimensional (2D) domain of size 80 by $50 \mathrm{Mm}$ (in $x-y$ ). We initialize with a linear force-free magnetic field given by

$$
\begin{gathered}
B_{x}=-B_{0} \cos \left(\frac{\pi x}{L_{0}}\right) \sin \theta_{0} \exp \left(-\frac{\pi y \sin \theta_{0}}{L_{0}}\right), \\
B_{y}=B_{0} \sin \left(\frac{\pi x}{L_{0}}\right) \exp \left(-\frac{\pi y \sin \theta_{0}}{L_{0}}\right), \\
B_{z}=-B_{0} \cos \left(\frac{\pi x}{L_{0}}\right) \cos \theta_{0} \exp \left(-\frac{\pi y \sin \theta_{0}}{L_{0}}\right),
\end{gathered}
$$

with the angle $\theta_{0}=30^{\circ}$ between the arcade and the neutral line $(x=0, y=0)$ and the horizontal size of our domain setting $L_{0}=80 \mathrm{Mm}$. The differences from Fang et al. (2015) is that we adopt a higher magnetic field strength of $B_{0}=80 \mathrm{G}$, instead of $50 \mathrm{G}$ in Fang et al. (2015), in order to have a realistic flare loop magnetic field as deduced from observations. For the initial thermal structure, we set a uniform temperature of $10,000 \mathrm{~K}$ below a height of $2.7 \mathrm{Mm}$ and fix the temperature variation to ensure a constant vertical thermal conduction flux $\kappa \partial T / \partial y=2 \times 10^{5} \mathrm{erg} \mathrm{cm}^{-2} \mathrm{~s}^{-1}$ above this height, identical to Fang et al. (2015). The initial density is derived from hydrostatic equilibrium with a number density of $1.2 \times 10^{15} \mathrm{~cm}^{-3}$ at the bottom. There is no flow to start with. To obtain a self-consistent thermally structured corona, we augment this setup with a background heating rate decaying exponentially with height, $H_{0}=c_{0} \exp \left(-\frac{y}{\lambda_{0}}\right)$ where $c_{0}=10^{-4} \mathrm{erg} \mathrm{cm}^{-3} \mathrm{~s}^{-1}$ and $\lambda_{0}=80$ $\mathrm{Mm}$. This heating appears as a source term in the energy equation, and is meant to balance the radiative losses and anisotropic heat conduction related losses of the corona in its equilibrium state. With this initial setup, we integrate the governing MHD equations until the above configuration reaches a quasi-equilibrium state at 144 minutes after initialisation, when we reset time to zero. In the remainder of this paper, we only discuss the stage following this relaxation phase. The relaxed

state of the system is reached when the maximal residual velocity is less than $10 \mathrm{~km} \mathrm{~s}^{-1}$ in the entire domain. Panel (a) and (b) in Fig. 1 show the number density and temperature of the relaxed system, respectively. The right frame (c) quantifies the corresponding thermal X-ray emission as explained further on, which is completely absent for this relaxed initial state. We use the MPIparallelized Adaptive Mesh Refinement Versatile Advection Code MPI-AMRVAC (Keppens et al. 2012; Porth et al. 2014; Keppens \& Porth 2014) with an effective resolution of $1024 \times 640$ or an equivalent spatial resolution of $79 \mathrm{~km}$ in both directions, obtained through four adaptive mesh refinement levels. 


\subsection{Triggering Chromosphere Evaporation}

The effect of the solar flare is modeled by its chromospheric energy deposit, handled as a finite duration heat pulse located at the loop footpoints. The temporal evolution of the heat pulse is controlled by $f(t)$, a piecewise linear ramping function to add and complete the sudden heating within 180 seconds. The asymmetric energy input is handled by a function $g(x)$, which sets the ratio of energy deposition at the left footpoint $(x<0)$ to the right footpoint $(x>0)$ to 0.8. Both functions appear in the heat pulse functional form $H_{1}(x, y, t)$ (which is an extra energy source term in addition to the background heating $\left.H_{0}(x, y)\right)$ specified as

$$
\begin{gathered}
H_{1}=c_{1} \exp \left(-\left(y-y_{c}\right)^{2} / \lambda^{2}\right) f(t) g(x) \\
\text { if } A\left(x_{1}, 0\right)<A(x, y)<A\left(x_{2}, 0\right) \\
A(x, y)=\frac{B_{0} L_{0}}{\pi} \cos \left(\frac{\pi x}{L_{0}}\right) \exp \left(-\frac{\pi y \sin \theta_{0}}{L_{0}}\right), \\
f(t)= \begin{cases}t / 30, & 0 \leq t<30 \mathrm{~s} \\
1, & 30 \leq t<150 \mathrm{~s} \\
(180-t) / 30, & 150 \leq t<180 \mathrm{~s}\end{cases} \\
g(x)= \begin{cases}1, & x>0 \\
0.8, & x<0\end{cases}
\end{gathered}
$$

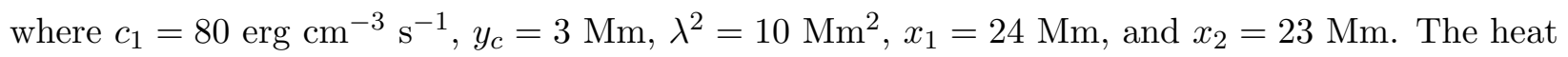
pulse is located close to the loop footpoint, i.e. $\approx 0.3 \mathrm{Mm}$ above the transition region, mimicking the footpoint heating by dissipated non-thermal particles. The energy of $H_{1}$ is expected to be quickly transported by thermal conduction away from the footpoints. The extra heating $H_{1}$ is active at two footpoints of a single magnetic loop or flux tube consisting of the magnetic field lines identified by the out-of-plane magnetic vector potential component $A(x, y)$ in the range of $x_{1}<|x|<x_{2}$.

\subsection{Estimating the thermal X-ray emission}

We estimate the thermal X-ray emission in the $3-12 \mathrm{keV}$ photon energy range based on the spatial distributions of density and temperature obtained from the simulation by the method described in Pinto et al. (2015) and also implemenetd in the open source forward modelling code FoMo available at https://wiki.esat.kuleuven.be/FoMo. The corresponding continuum thermal X-ray photon flux density of a fully ionized hydrogen plasma emitted at the photon energy $h \nu$ is defined as

$$
\begin{gathered}
I(h \nu, T)=I_{0} \frac{\mathrm{EM}}{h \nu \sqrt{k_{b} T}} g_{f f}(h \nu, T) \exp \left(-\frac{h \nu}{k_{b} T}\right), \\
g_{f f}(h \nu, T)=\left\{\begin{array}{ll}
1, & h \nu \lesssim k_{b} T \\
\left(\frac{k_{b} T}{h \nu}\right)^{0.4}, & h \nu>k_{b} T
\end{array},\right.
\end{gathered}
$$


where EM is the emission measure $n^{2} V$ of a finite volume $V$ of plasma of number density $n$ and temperature $T$, the coefficient $I_{0}$ is $1.07 \times 10^{-42}$ for a photon flux measured at a distance of 1 $\mathrm{AU}$, if the photon flux density is expressed in units of photons $\cdot \mathrm{cm}^{-2} \cdot \mathrm{s}^{-1} \cdot \mathrm{keV}^{-1}$, and $g_{f f}(h \nu, T)$ is the Gaunt factor for free-free bremsstrahlung emission. We compute the photon flux density at different photon energies for each individual grid cell, and then integrate the total photon flux density as $D N=\sum I(h \nu, T) \Delta h \nu$ in the $3<h \nu<12 \mathrm{keV}$ photon energy range. We use $0.01 \mathrm{keV}$ as $\Delta h \nu$, meaning totally 90 bins in the $3<h \nu<12 \mathrm{keV}$ energy range. This process leads to the figures shown in Fig. 1, right column.

\section{Results and Discussion}

\subsection{Asymmetric footpoint heating inducing KHI}

The temporal evolution of the number density (left column), temperature (middle column), and synthesized SXR photon fluxes (right column) are shown in Fig. 1, covering a timespan of about 4.15 minutes. As described in $\S 2.2$, we use $H_{1}$ to mimic the flare energy deposited in the upper chromosphere, and the consequent overpressure resulting from $H_{1}$ drives upward mass flows at a speed up to $700 \mathrm{~km} \mathrm{~s}^{-1}$. We also witness a rapid thermodynamic change (due to thermal conduction) in the loop, reaching an extremely hot temperature around $18 \mathrm{MK}$. These upward chromospheric evaporation flows have been detected directly in imaging sequences as SXR emission propagating toward the loop-top sources (Silva et al. 1997; Liu et al. 2006; Nitta et al. 2012). This is also seen in our simulation, as in panel (f) of Fig. 1 (and in the time period before this image, for which we refer to the corresponding movie). In the last several decades, simulations of chromospheric evaporation were mostly carried out with symmetric energy input assumptions on both loop footpoints and these focused mainly on the UV-SXR spectral lines produced by the evaporated plasma (Fisher et al. 1985; Mariska et al. 1989; Yokoyama \& Shibata 2001; Allred et al. 2005; Liu et al. 2009). However, asymmetric energy deposition at footpoints is a much more likely scenario, and could be intrinsic to the acceleration process as pursued by McClements \& Alexander (2005). So, in our simulation we assume that the deposition of flare energy at two footpoints is slightly asymmetric, with a ratio of 0.8 as described in equation (5). As a result, the right evaporation flow rises quicker and is hotter than the left one as shown in panel (e). The asymmetric energy deposition guarantees that the two evaporation flows will not merely collide at the apex, a situation known to only generate reflected shocks. Panel (e) indeed shows that when our two evaporation flows run into each other asymmetrically, the right one has already crossed the loop apex. In addition, the hotter, right evaporation flow causes a bigger expansion of the loop cross-section in the right half of the loop. This configuration provides the conditions for the formation of shear flows, the trigger of the KHI. As shown in panel (e), right after the collision of both flows, the KHI begins to develop immediately, and the typical vortical structures of the KHI appear and grow up quickly from panel (e) to (g). The development of vortices in a magnetized plasma can further induce small-scale structure to develop, with a potential cascade of energy to 
establish a turbulent plasma state (Keppens et al. 1999; Baty et al. 2003; Elmegreen \& Scalo 2004; Henri et al. 2013).

Our grid-adaptive simulations offer enough resolution to also capture a number of (numerical) reconnection processes occurring during the vortex development. The left column in Fig. 2 shows the temporal evolution of the current distribution perpendicular to the plane (the component $J_{z}$ ) in a color scale, overlaid with contours of the magnetic vector potential which identify magnetic field lines. By the vortical winding up of the field, we obtain locally anti-parallel magnetic field lines and accompanying strong current sheets, all introduced by the KHI as a trigger for local reconnection processes near the apex as shown in panel (c). The resulting magnetic islands keep evolving, merging, and splitting for the following 4 minutes. In fully kinetic simulations of isolated reconnection layers, Drake et al. (2006) demonstrated that the formation of micro-turbulence inside current sheets can act as a non-thermal particle accelerator. Although we only simulated the MHD scales, the mesoscopic (i.e. in between the macro-scale of the loop diameter and the kinetic scales) current sheets in panel (c) have the possibility to form micro-turbulence inside them and accelerate particles very efficiently. Normally, in an open magnetic configuration, such high energy particles escape easily in the order of seconds. In that case, the HXR emission which depends on the density of non-thermal particles cannot achieve a high intensity. However, in our simulation, the accompanying rotating magnetic islands exist on MHD scales for several minutes, so they can trap the accelerated particles during their formation at the loop apex. Note that the local island structures indeed have minute-long lifetime and fine-structure variation, which matches the time scale and variation suggested by the observations for loop-top HXR sources (Krucker et al. 2008). The observations suggest that the time variation of loop-top HXR sources are most prominent during the rise of the thermal emission, normally observed as SXR emission (Krucker et al. 2008). In order to confirm that the chromospheric evaporation flows not only have the ability to trigger the KHI to establish the preferred looptop site for particle acceleration, but also provide enough SXR photons for the Inverse Compton mechanism producing HXR emission, we calculate the thermal emission of the plasma by the method in $\S 2.3$ in the energy range of $3-12 \mathrm{KeV}$, a typical SXR band in RHESSI, as shown by the right column in Fig. 1. At the initial stage of the flare energy deposition, the SXR emission is concentrated at the two footpoints, and then rises to the apex. Afterwards, the collision between two evaporation flows compresses the plasma at the loop-top area and increases both the number density and temperature there, resulting in a bright loop-top SXR source as shown in panel (f), (i) and (l). The evolution of SXR emission in our simulation not only recovers the previous observational result of SXR emission in flare events (Liu et al. 2006; Veronig et al. 2005), but also indicates the co-spatial and simultaneous relationship between the particle accelerator introduced by the KHI and the generation of SXR photons from thermal emission of hot plasma. Thus, the Inverse Compton emission mechanism has all ingredients to create a HXR source with time order of minutes at the apex. 


\subsection{Ingredients for non-thermal particle acceleration}

The exact nature of particle acceleration in flares is still a matter of debate (Fletcher \& Hudson 2008; Brown et al. 2009), but our simulation supports the suggestion by Krucker et al. (2008) that loop-top non-thermal particles are very likely accelerated and trapped by localised turbulent plasma. Turbulence is the most natural agent for establishing an energy cascade over a large dynamical range, and the corresponding stochastic acceleration models have been widely used for solar flare studies (Miller 1997; Petrosian \& Liu 2004). We find strong indications of KHI induced turbulence at the apex in our simulation. Such turbulence could be a possible accelerator for non-thermal particles. The acceleration mechanism in turbulence is usually first order Fermi acceleration by shocks and second order Fermi acceleration by stochastic processes (Fermi 1949). From our MHD model, we can only argue how shocks and stochastic processes are indeed locally realized. Panel (a) in Fig. 3 shows the absolute value of the divergence of the velocity field in the zoomed-in region shown by the white rectangle in panel (c) of Fig. 1. This identifies the locations of strong compression, an indication of shock fronts, and suggests that first order Fermi acceleration can take place efficiently there. We also show the flow field itself in panel (b), showing that the island structures seen in Fig. 2 are indeed correlated to vortical flow structures. We interpret the fine-structure seen as (not yet fully resolved) turbulence which infers that stochastic acceleration could also play a part in this region as well.

The efficiency of the acceleration process in turbulence strongly depends on the existence of short wavelength structures (or high wave number components). We perform a spatial Fourier analysis on each frame of $J_{z}(x, y)$ shown in the left column of Fig. 2, by forward Fast Fourier Transform procedure (FFT, IDL8.2). With shifting the zero-frequency component to the center of the spectrum, FFT governs the equation as

$$
F\left(k_{x}, k_{y}\right)=\frac{1}{N M} \sum_{x=-N / 2}^{N / 2} \sum_{y=-M / 2}^{M / 2} J_{z}(x, y) \exp \left(-i 2 \pi\left(\frac{k_{x} \cdot x}{N}+\frac{k_{y} \cdot m}{M}\right)\right),
$$

which implies the zero point symmetry in wave number domain. We then obtain the corresponding log-scaled power spectrum in the wave number domain in the right column of Fig. 2. Before the start of KHI at $t \approx 41$ seconds, panel (b) shows that the distribution of this power spectrum is more centrally peaked at the low wave numbers, in accord with non-existence of small structures then. During the development of the KHI at $t \approx 249$ seconds, panel $(\mathrm{d})$ demonstrates that the distribution of power spectrum spreads out to the larger wave numbers, indicating that smaller scale structures are generated. After the fading of the shear flows and vortices at $t \approx 369$ seconds, the KHI are suppressed by the magnetic field, and panel (f) displays that the power spectrum contracts back into the small wavenumber regime again, meaning that the small structures disappear. The evolution of the power spectrum in the right column of Fig. 2 reveals that the KHI does introduce strong turbulence during its evolution at the apex. 


\section{Discussion and conclusion}

In this letter, we propose a new ingredient to the standard model for solar flares, for the rare events where one witnesses the formation of loop-top HXR sources. Our model provides further support to the interpretation that these HXR loop-top sources can occur through the Inverse Compton mechanism scattering the surrounding SXR photons to higher energy. We show that a slight asymmetry between the left-right footpoint deposited energy by non-thermal particles from the reconnection layer developing above the loops is sufficient to establish rapid chromospheric evaporation flows with speeds up to $700 \mathrm{~km} / \mathrm{s}$ that are liable to Kelvin-Helmholtz instability (KHI) when they meet near the loop apex. As usual in KHI evolutions in magnetized plasma conditions, vortices form further fine scale structure within, and the turbulence that appears at the apex of loops could be considered as an efficient accelerator for non-thermal particles. The island structures are ideal sites for trapping non thermal particles at the loop-top. We show that our model reproduces the thermal SXR evolution related to the evaporation flows, as well as establishing a pronounced SXR source region in the loop-top. We suggest that locally accelerated non-thermal particles can easily upscatter these thermal SXR photons to HXR photons. This needs to be further investigated by augmenting the MHD scenario demonstrated here, with test particle evolutions to quantify the

efficiency of the acceleration process. Our work provides strong support to the role of KHI as a clear trigger of loop-top SXR to HXR emission, during the impulsive phase of single loop flares.

The research has been sponsored by an Odysseus grant of the FWO Vlaanderen. The results were obtained in the KU Leuven GOA project GOA/2015-014 and by the Interuniversity Attraction Poles Programme initiated by the Belgian Science Policy Office (IAP P7/08 CHARM). Part of the simulations used the infrastructure of the VSC - Flemish Supercomputer Center, funded by the Hercules Foundation and the Flemish Government - Department EWI. We acknowledge the fruitful discussion with Hugh Hudson, Yang Guo and Patrick Antolin.

\section{REFERENCES}

Allred, J. C., Hawley, S. L., Abbett, W. P., \& Carlsson, M. 2005, ApJ, 630, 573

Andries, J., \& Goossens, M. 2001, A\&A, 368, 1083

Baty, H., Keppens, R., \& Comte, P. 2003, Physics of Plasmas, 10, 4661

Berger, T. E., Slater, G., Hurlburt, N., et al. 2010, ApJ, 716, 1288

Brown, J. C., Turkmani, R., Kontar, E. P., MacKinnon, A. L., \& Vlahos, L. 2009, A\&A, 508, 993

Brown, J. C. 1971, Sol. Phys., 18, 489

Chandrasekhar, S. 1961, International Series of Monographs on Physics, Oxford: Clarendon, 1961,

Drake, J. F., Swisdak, M., Che, H., \& Shay, M. A. 2006, Nature, 443, 553

Elmegreen, B. G., \& Scalo, J. 2004, ARA\&A, 42, 211 
Fang, X., Yuan, D., Van Doorsselaere, T., Keppens, R. \& Xia, C. 2015, ApJ, 813, 33

Fermi, E. 1949, Physical Review, 75, 1169

Fisher, G. H., Canfield, R. C., \& McClymont, A. N. 1985, ApJ, 289, 414

Fletcher, L. 1995, A\&A, 303, L9

Fletcher, L., Dennis, B. R., Hudson, H. S., et al. 2011, Space Sci. Rev., 159, 19

Fletcher, L., \& Hudson, H. S. 2008, ApJ, 675, 1645

Foullon, C., Verwichte, E., Nakariakov, V. M., Nykyri, K., \& Farrugia, C. J. 2011, ApJ, 729, L8

Frost, K. J., \& Dennis, B. R. 1971, ApJ, 165, 655

Heyvaerts, J., \& Priest, E. R. 1983, A\&A, 117, 220

Henri, P., Cerri, S. S., Califano, F., et al. 2013, Physics of Plasmas, 20, 102118

Hoyng, P., Duijveman, A., Machado, M. E., et al. 1981, ApJ, 246, L155

Hudson, H. S. 1978, ApJ, 224, 235

Hudson, H. S. 2011, Space Sci. Rev., 158, 5

Keppens, R., Tóth, G., Westermann, R. H. J., \& Goedbloed, J. P. 1999, Journal of Plasma Physics, 61, 1

Keppens, R., Meliani, Z., van Marle, A. J., Delmont, P., Vlasis, A., \& van der Holst, B. 2012, JCP, 231, 718

Keppens, R. \& Porth, O. 2014, Journal of Computational and Applied Mathematics, 266, 87

Korchak, A. A. 1971, Sol. Phys., 18, 284

Krucker, S., \& Lin, R. P. 2008, ApJ, 673, 1181

Krucker, S., Battaglia, M., Cargill, P. J., et al. 2008, A\&A Rev., 16, 155

Krucker, S., \& Battaglia, M. 2014, ApJ, 780, 107

Liu, W., Liu, S., Jiang, Y. W., \& Petrosian, V. 2006, ApJ, 649, 1124

Liu, W., Petrosian, V., \& Mariska, J. T. 2009, ApJ, 702, 1553

Mariska, J. T., Emslie, A. G., \& Li, P. 1989, ApJ, 341, 1067

Masuda, S., Kosugi, T., Hara, H., Tsuneta, S., \& Ogawara, Y. 1994, Nature, 371, 495

McClements, K. G., \& Alexander, D. 2005, ApJ, 619, 1153

Miller, J. A. 1997, ApJ, 491, 939

Neupert, W. M. 1968, ApJ, 153, L59

Nitta, S., Imada, S., \& Yamamoto, T. T. 2012, Sol. Phys., 276, 183

Petrosian, V., \& Liu, S. 2004, ApJ, 610, 550

Pinto, R. F., Vilmer, N., \& Brun, A. S. 2015, A\&A, 576, A37

Porth, O., Xia, C., Hendrix, T., Moschou, S. P., \& Keppens, R. 2014, ApJS, 214, 4

Shibata, K., \& Magara, T. 2011, Living Reviews in Solar Physics, 8, 6 
Silva, A. V. R., Wang, H., Gary, D. E., Nitta, N., \& Zirin, H. 1997, ApJ, 481, 978

Tomczak, M. 2001, A\&A, 366, 294

Turkmani, R., Cargill, P. J., Galsgaard, K., Vlahos, L., \& Isliker, H. 2006, A\&A, 449, 749

Veronig, A. M., \& Brown, J. C. 2004, ApJ, 603, L117

Veronig, A. M., Brown, J. C., Dennis, B. R., et al. 2005, ApJ, 621, 482

Yokoyama, T., \& Shibata, K. 2001, ApJ, 549, 1160

This preprint was prepared with the AAS LATEX macros v5.2. 

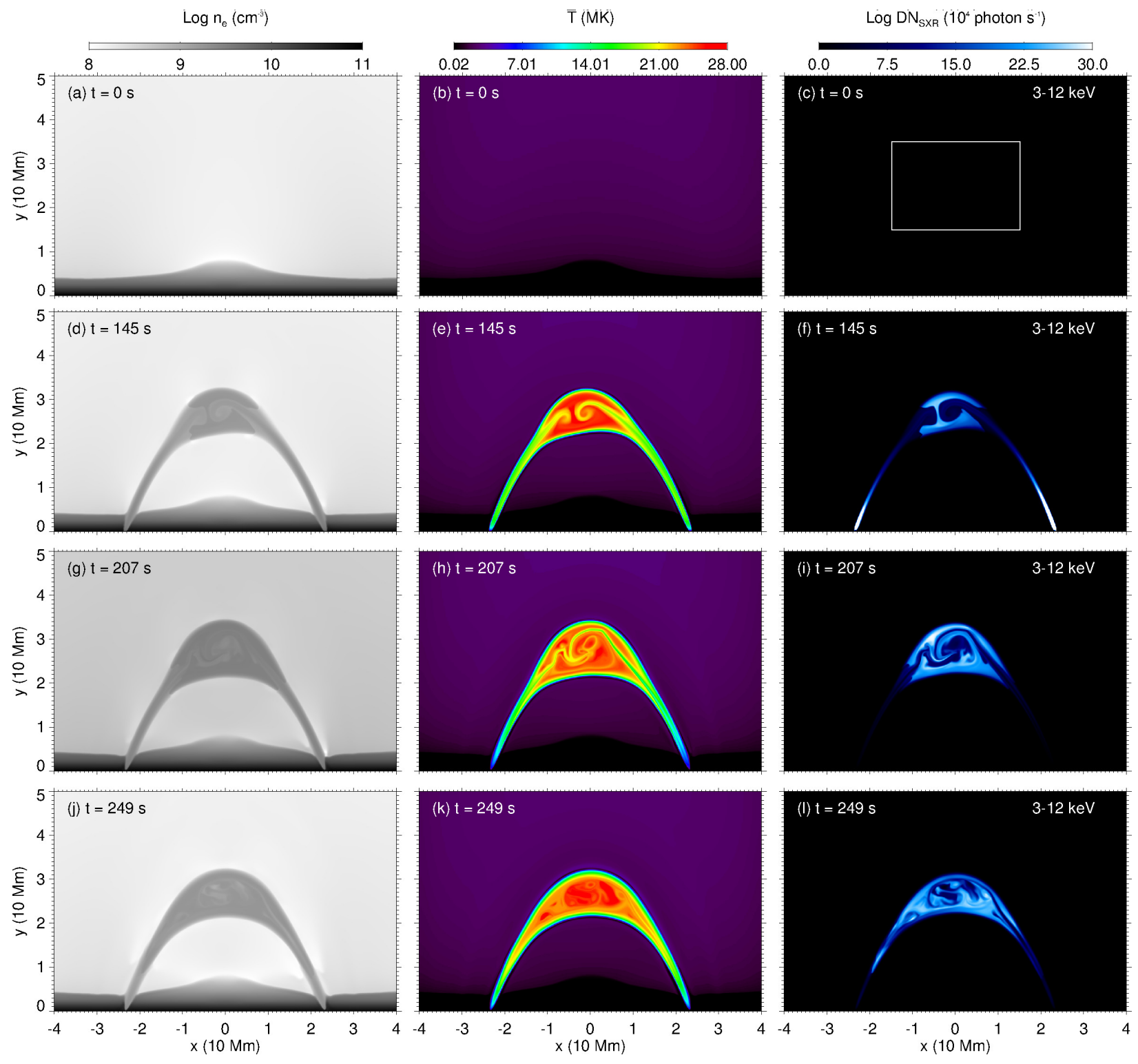

Fig. 1. - Temporal evolution of number density (left column), temperature (middle column), and synthesized SXR photon flux images (right column) at $t \approx 0,145,207$ and 249 seconds, respectively. There is an animation (m1.mp4) of Fig. 1 available online. 

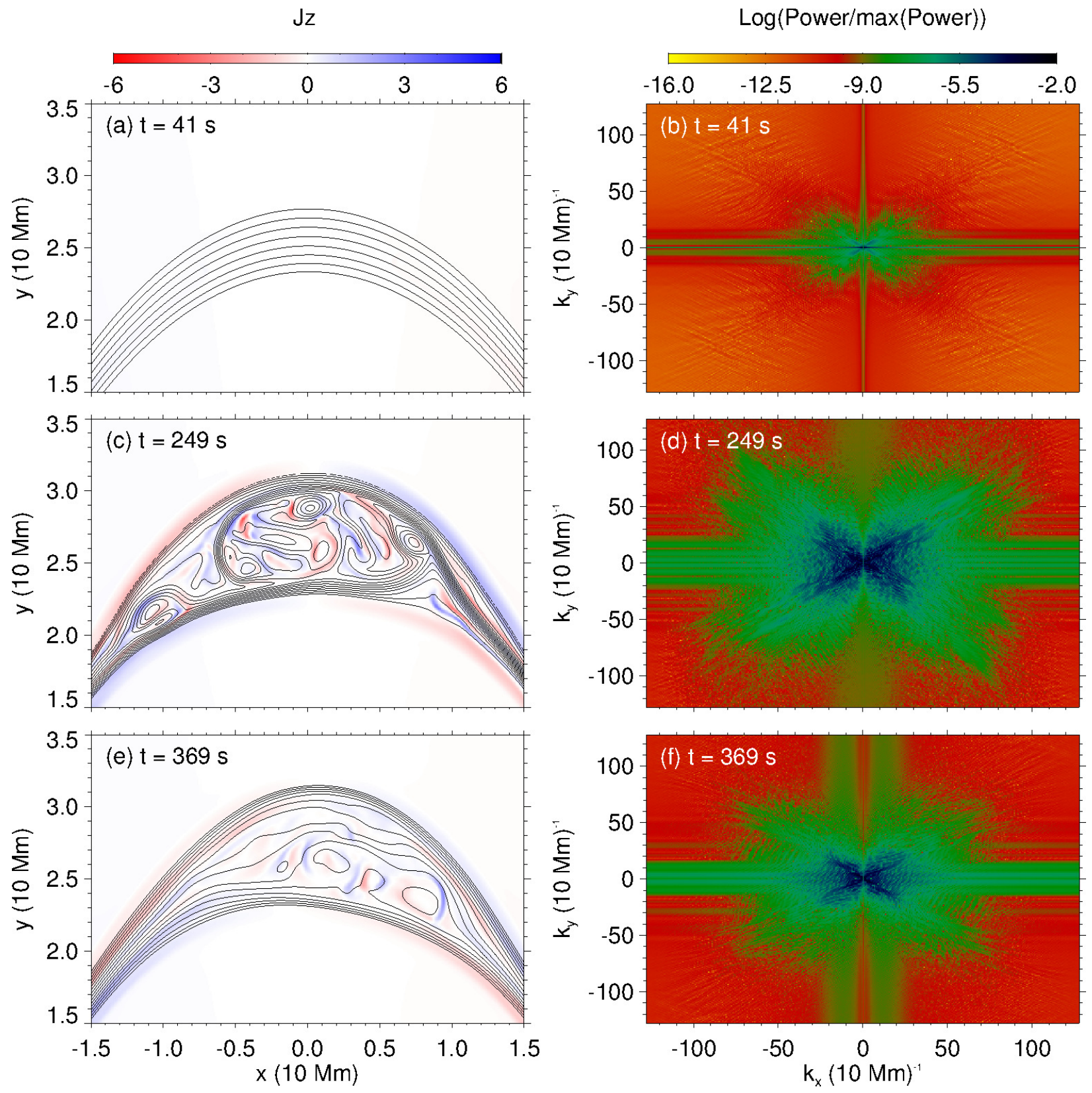

Fig. 2.- For the region in the white rectangle in panel (c) of Fig. 1, the temporal evolution of the current $J_{z}$ perpendicular to the plane, overlaid by contours of magnetic vector potential (left column), and log-scaled power spectrum from Fourier analysis of the $J_{z}$ images (right column) at $t \approx 41,249$, and 369 seconds, respectively. 

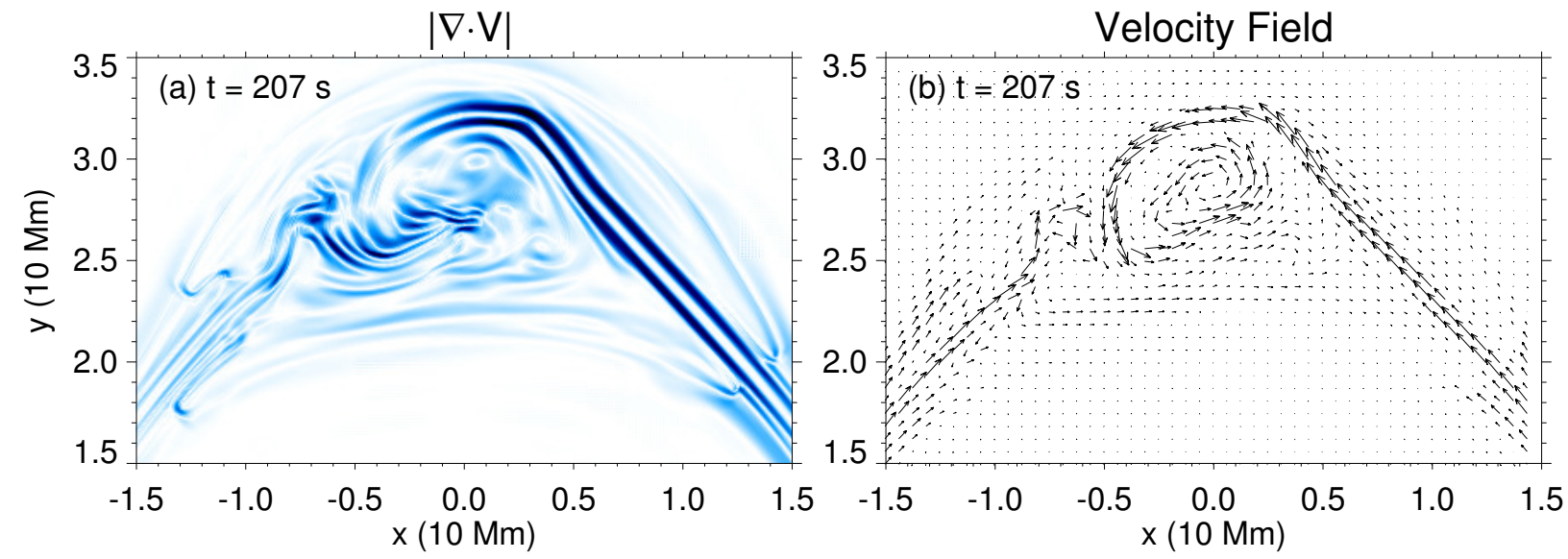

Fig. 3. - At $t \approx 207$ seconds, inside the region shown by the white rectangle in panel (c) of Fig. 1 , we show in panel (a) the absolute value of the divergence of the plasma velocity and the flow field itself in panel (b). 